\title{
An evolutionary hypothesis for obsessive compulsive disorder: a psychological immune system?
}

\author{
Riadh T. Abed ${ }^{\mathrm{a}, *}$ and Karel W. de Pauw ${ }^{\mathrm{b}}$ \\ ${ }^{\mathrm{a}}$ Rotherham District General Hospital, Moorgate \\ Road, Rotherham S60 2UD, UK \\ ${ }^{\mathrm{b}}$ St James's University Hospital, Leeds LS9 7TF, UK
}

\begin{abstract}
A new hypothesis is presented within the framework of evolutionary psychology that attempts to explain the origins of obsessivecompulsive disorder. It is suggested that obsessions and compulsions originate from the overactivity of a mental module that the majority of humans possess and has the function of generating risk scenarios without voluntary intervention. It is hypothesised that obsessional phenomena function as an off-line risk avoidance process, designed to lead to risk avoidance behaviour at a future time, thus distinguishing it from anxiety and related phenomena as on-line emotional states, designed to lead to the avoidance of immediate and direct risks. Finally, the hypothesis makes a number of specific predictions that are testable and refutable. It is contended that the present hypothesis if supported by empirical evidence could serve as a basis for future research on this important disorder.
\end{abstract}

Keywords: Darwinism, evolutionary psychology,

obsessive-compulsive disorder, post-traumatic stress disorder

\section{Introduction}

Evolutionary theory recognises causation at ultimate and proximate levels. Ultimate causation refers to the supposed function of an adaptation or organ within the ancestral environment while proximate causes refer to all the possible physical factors that directly produce the given trait (e.g., biochemical, genetic, etc.). The present hypothesis attempts to give an account of the ultimate causation of obsessivecompulsive disorder (OCD). It is based upon the Darwinian view that biological systems, including psychological functions, have evolved through natural selection because of their contribution to inclusive fitness [23]. The following hypothesis attempts to give an account of the normal (adaptive) functioning of the neu-

${ }^{*}$ Corresponding author: Riadh T. Abed; E-mail: abed@globalnet.co.uk. robiological system responsible for obsessional phenomena, an area that has hitherto received scant attention in the psychiatric and psychological literature.

Darwinian theorists have suggested that some idiopathic psychiatric symptoms and syndromes may represent misplaced adaptive strategies or accentuated versions of original strategies which can be caused by a number of proximate factors (see $[1,8,31,32,33,35$, 50]). It is the contention of this hypothesis that OCD is an accentuated version of an adaptive strategy that enhanced the reproductive fitness of those humans who possessed this trait over those who did not within their ancestral environment. The fact that the experience of unwanted intrusive thoughts and compulsive rituals are universal phenomena across cultures is consistent with this view [36, 44]. Furthermore, the relatively high lifetime prevalence of OCD of around $2.5 \%$ and one-year prevalence of $1.6 \%$ [26] would argue against the condition being caused by a harmful genetic mutation but is consistent with it representing the severe end of a potentially adaptive trait [53], instead.

Neurobiological systems have evolved to generate adaptive psychological or behavioural strategies and not to produce psychopathological states [22]. It should therefore follow that dysfunction (or apparently disordered functioning) cannot be clearly conceptualised until the proper function of a biological system is correctly identified (see $[5,8]$ ).

It has often been the case that pathological changes within the nervous system reveal psychological functions that had hitherto remained unknown [6]. Examples of pathological states that have produced insights into the functioning of certain neurobiological brain systems are many and include dysphasia, dyspraxia and the frontal lobe syndrome, to name but a few.

The present formulation assumes a modular configuration for the human mind/brain [11, 15, 20, 38, 48]. Modularity suggests that the human mind consists of a number of domain-specific, highly tuned, systems that have evolved to carry out circumscribed tasks. 
Such tasks would have been selected because of their contribution to inclusive fitness.

This contrasts with the alternative view that is prevalent within the social sciences of the human brain as a domain-free general-purpose problem solving device. The current hypothesis also concurs with the suggested modular configuration of the brain system concerned with obsessional phenomena proposed by [44].

\section{Hypothesis}

It is contended that obsessional phenomena are archaic, involuntary, repetitive thought processes that stimulate strong aversive emotional states (e.g., fear, disgust) and lead to risk avoidance behaviour. It is hypothesised that the neurobiological system that generates these phenomena has the function of generating risk scenarios without conscious intervention and may thus function as an 'Involuntary Risk Scenario Generating System' (IRSGS). Compulsive rituals, the other component of OCD, are conceptualised as primitive harm avoidance behavioural routines that are under semi-voluntary control [6]. It is suggested that the IRSGS operates primarily as a self-generated conditioning system whereby the individual can develop harm avoidance behavioural strategies without experiencing the risks involved in real-life dangers.

The adaptive function of this system is that it saves the individual organism from having to experience physical and social dangers in vivo, but instead produces the same learning response in total physical safety. Therefore, the ability of some organisms to learn to avoid common dangers without the need to experience them in real life would have conferred a clear advantage on the individuals who possessed this trait over those who did not. This would have ensured the spreading of the obsessional traits within the population but, as with most traits, extreme variants may prove harmful and can reduce reproductive fitness.

The obsessional process may be akin to the generation of antibodies by the immune system. Whether or not an antibody increases in concentration will depend upon the subsequent feedback. While antibodies have the function of protecting the body from invisible dangers within the body, obsessional thoughts are designed to protect the individual primarily from external dangers within the environment. It is suggested that both operate through a selectionist process - that is, the better fit antibody or thought survives. Some authors have suggested that a similar process, involv- ing the selection of random and spontaneously generated thoughts, lies at the heart of all human thinking [4, 21, 40, 41].

Bickerton [3] has proposed that there are two fundamental modes of thinking: on and off-line. On-line thinking is common to many complex organisms and involves mental activity designed to solve a problem directly faced by an individual. Off-line thinking involves mental activity aimed at solving problems that the organism may face at some time in the future. Bickerton contends that off-line thinking is language-based and, therefore, unique to the human species. Within this framework, obsessional thinking could be considered as a primitive variant of off-line thinking, albeit of a non-voluntary type. Although the idea that human thought is language based is not universally accepted [38], there may be grounds to suggest that obsessional phenomena could have been the evolutionary precursor to voluntary conscious thinking. This would raise the interesting question as to whether obsessions, as conceptualised in this paper, exist in other species. The main obstacle in studying such a system in nonhumans is, of course, the inaccessibility of their subjective states and, at least at present, the question can only be investigated indirectly through determining if something akin to obsessions could be implicated in learning in other species.

Most current animal models of OCD are based on the study of maladaptive, repetitive behaviours (e.g., [17]). Such behaviour is thought to represent built-in survival actions encoded in the basal ganglia and arising due to dysfunction in these brain centres. Similarly, compulsions and tics in humans have been considered as fragments of once purposeful activities that arise inappropriately due to dysfunction within the cortico-striatal system [10]. While of great interest the scope of these models does not extend to the phenomena of obsessional thoughts. Nevertheless, the suggestion of an innate, cross-species neurobiological system, charged with the generation of vital (primarily harm avoidance) survival behaviours, is obviously consistent with the current hypothesis.

Ratey and Johnson [45] make an important distinction between social and non-social 'scanners' among OCD patients. They contend that OCD patients tend to persistently scan their environment for sources of risk and that it is possible to separate those who tend search their environment for social dangers from those who anticipate physical risks (e.g., contamination). Marks and Nesse [32] have similarly drawn attention to a number of physical and social risk-prevention strate- 
gies commonly involved in obsessional phenomena. Hence, given that human reasoning about the social domain is claimed to involve distinct modular brain processing $[2,15,34]$, the IRSGS may process risk scenarios concerning physical and social situations separately. In other words, it is suggested here that the obsessional phenomenon is a self-generated conditioning process, directed at two distinct and separate domains namely the physical and the social environments.

The present formulation assumes that obsessions and compulsions arise from a psychological adaptation that the majority of humans possess. This is consistent with reports that a majority of the population experience unwanted, intrusive thoughts at some time during their lives $[36,42]$ as well as the finding that OCD appears to have a universal form across cultures $[44,49]$.

The subject of obsessive-compulsive features as part of normal child development has received some attention recently [51]. For example, ritualised behaviour is quite common in children at around 30 months of age, appears to decline at the age of three and tends to disappear at around four when the child becomes thoroughly familiar with his or her surroundings. Furthermore, it has been suggested that the difference between normal children and the minority who later develop OCD may lie in the degree to which the latter were controlled and disabled by their thoughts rather than any other specific clinical or developmental features [27].

Further investigation will be required to discover the details of the natural development of obsessional phenomena e.g., whether or not those pertaining to the physical and social domains have distinct developmental histories. Furthermore, it could be predicted that gender differences would exist, both in the developmental history of obsessions as well as periods of maximum risk of onset, as males and females would have faced distinct environmental and social risks during evolutionary history.

It has already been suggested that the neurobiological system responsible for generating obsessional thoughts is the mental or psychological analogue to the immune system. Rather than being responsible for the production of protective proteins (antibodies) that neutralise physical danger from potential invaders, the IRSGS would be charged with the production of risk scenario packages that use elements of the physical and social environment to predict certain dangers. The accompanying negative emotions of anxiety, disgust or fear should result in avoidance behaviour and a process of learning in imagery which usually leads to the individual becoming 'immunised.' At this point the random danger scenarios should cease or simply become dormant. Thus if the present hypothesis is correct, OCD would be analogous to a mental auto-immune disease, i.e., a protective response that goes beyond the point of usefulness and becomes selfdestructive.

From a Darwinian point of view, both physical and social risks can be conceptualised as risks to self and/or close kin according to Hamilton's principle of inclusive fitness [23]. Therefore, obsessional thoughts will have content that relates to dangers or risks to self or kin (or kin substitutes) in either of these two domains, namely the social and the physical. The nature and variety of risks would be selected from the array of stimuli that exists within the individual's environment. However, the process through which such dangers or risks are identified remains at present unknown. We are equally ignorant as to how a particular ritual or compulsive activity is selected from the array of possible alternatives. If obsessive-compulsive phenomena relate to risk scenarios to self and close kin, it should be possible to predict an increase in the frequency and intensity of risk scenarios and harm avoidance rituals at biologically critical times, e.g., following childbirth or around puberty. Current evidence supports the claim that pregnancy and childbirth are associated with significantly increased risk of OCD for women [9].

The phenomenological similarities between OCD and post-traumatic stress disorder (PTSD) have been noted by a number of authors (e.g., [28]). According to the proposed hypothesis, the frequency of obsessional thoughts would be expected to increase following traumatic or threatening situations, whether involving physical danger or distressing social situations. Such events should therefore activate the IRSGS by presenting the subject with a range of novel risk situations and provide a potential explanation for at least some of the clinical features of PTSD e.g., recurrent intrusive thoughts and images as well as recurrent dreams. Therefore, from an evolutionary perspective PTSD, could be considered as a pathological accentuation of an adaptive response that previously led to the modification of an individual's behaviour and future risk reduction (i.e., learning). In addition to thoughts and images during the waking state, the IRSGS may also utilise dreams as a mechanism for risk avoidance, thus accounting for the increased frequency of vivid dreams and nightmares characteristic of PTSD. How- 
ever, it is clear that these two disorders, i.e., OCD and PTSD, also differ in some important respects. For example the absence of compulsive rituals in PTSD cannot be explained within the present hypothesis.

It is contended, therefore, that anxiety and panic are on-line emotional states, designed to generate risk avoidance behaviour as a direct response to the exposure to immediate (real or imagined) dangers, whereas the obsessional system is an off-line process primarily designed to generate harm avoidance behaviour in response to dangers that may be encountered at some time in the future.

\section{Predictions}

A number of potentially testable and refutable predictions can be made, based upon the present hypothesis. These are:

1. Individuals with antisocial personality disorder (APD), especially those diagnosed as psychopathic according to the criteria of Cleckley $[12,24,30]$, will show a lower frequency of social content obsessional thoughts than the general population. The suggestion that individuals suffering from OCD or APD lie on opposite ends of the risk seeking and harm avoidant scale is consistent with this prediction [25].

2. Individuals who habitually engage in dangerous activities, including certain sports, should experience a lower incidence of obsessional phenomena directed at non social environmental dangers.

3. Obsessional patients should be more socially conforming than the average person and less prone to risk taking. There are a number of reports that suggest that this is the case [36]. It would be of interest to determine whether OCD patients divide into social conformists as opposed to low physical risk takers.

4. A clinical picture analogous to PTSD may result from a state of social trauma (e.g., failure, humiliation, disgrace).

5. The frequency of unwanted intrusive thoughts should be positively correlated with the score on the dimension of 'harm avoidance' of the seven factor personality system [14]. This prediction is consistent with the suggestion that OCD patients score highly on this dimension [13].

6. There should be an increased risk of OCD at different, biologically critical, life stages, e.g., during pregnancy and following childbirth, when a range of novel risks will become relevant to the subject. Similarly, an increased risk of OCD may be expected following bereavement, as normal grief is associated with an increase in the frequency of recurrent intrusive images and thoughts which may represent increased activity of the IRSGS.

\section{Discussion}

The present hypothesis is compatible with a range of other proposals and theories dealing with the proximate level of causation, i.e., genetic, neurochemical and environmental factors, in OCD. Psychological theories that emphasise an acquired environmental causation (e.g., [47]) as well as findings pointing to a biological aetiology of OCD [46] are both consistent with the present Darwinian formulation. Whereas the present hypothesis describes the adaptive function of a putative neurobiological system, addressing the question of 'why' it exists, it is compatible with theories that explain 'how' it works.

Hence, the present hypothesis presents a novel formulation to explain the ultimate causation of OCD, thus attempting to integrate this condition into the now well-established field of evolutionary psychology and psychiatry. An important feature of the current hypothesis is the proposal of a putative mental module (IRSGS) that is charged with the generation of risk scenarios without voluntary intervention which, in turn, give rise to a range of risk prevention behaviours described as compulsions. This proposal uniquely contends that overactivity of the IRSGS will lead, in certain circumstances, to OCD or PTSD while underactivity of this module may be associated with APD or other manifestations of persistent high risk-taking behaviour. Furthermore, the current hypothesis clearly proposes that obsessional phenomena are examples of a mental adaptation that has evolved through a process of selection and that OCD is, therefore, the result of the malfunctioning or dysregulation of this system.

Thus, conceptualising obsessions as risk scenarios and compulsions as risk prevention routines will help to clarify the clinical observation that while obsessions increase anxiety, compulsive rituals reduce anxiety (at least temporarily) [43].

Epidemiological surveys of OCD have found a small preponderance of female subjects [52]. As females would be expected to engage less frequently than males in risk taking behaviour [7, 16] such a finding would 
be consistent with the present hypothesis. Also there is evidence that gender differences vary according to the age of onset of OCD with males showing a higher prevalence prepubertally and females more commonly affected post-pubertally [37]. It is possible that the increased risks that females were exposed to following puberty within the ancestral environment could have led to the increased activity of the IRSGS leading to the greater incidence of OCD.

At present, however, it has to be acknowledged that there is a range of data that may be difficult to reconcile with certain aspects of the present hypothesis.

One such area is that of the co-existence of impulsivity and compulsivity that has been suggested by some authors (see [29]). If obsessional phenomena are part of a harm reduction system then the occurrence of impulsive (risk taking) behaviour would be difficult to reconcile with the present formulation. It would, however, be of interest to determine whether impulsivity and compulsivity show a greater probability of co-existing if they belong to different domains (i.e., the social as opposed to the physical domain).

Comparisons are frequently drawn between the symptoms of OCD and certain religious practices and it has been suggested that both may stem from the human propensity to produce socially meaningful rituals $[18,19]$. However, the present hypothesis would argue for a wider and more generically adaptive role for these phenomena which is not solely restricted to the maintenance of social cohesiveness alone.

As already indicated, the present hypothesis leaves a number of questions unanswered. For example, what is the relationship between the generation of risk scenarios and habituation? Is there a feedback mechanism? Does OCD arise when habituation fails or when the feedback mechanism fails, or perhaps both? Or does it simply arise through an intrinsic dysfunction of the IRSGS? Alternatively, as a clinical syndrome with a number of aetiologies, it may turn out to be the case that OCD represents a final common pathway, resulting from breakdowns in different systems or at different levels in one system.

The proposed model does not predict or specify the proximate neural or neurochemical systems that are instrumental in causing the overactivity of the putative IRSGS manifesting clinically as OCD. However, this formulation presents a plausible, albeit preliminary, account of the possible function of the underlying neurobiological system responsible for generating pathological obsessions. Science does not only advance through slicing, homogenising and measuring but also through the highly important and somewhat neglected activity of constructing conceptual models that generate testable hypotheses. Hence, if this proposal is supported by empirical evidence it may serve as a framework for generating further hypotheses and formulating questions that can guide future research into this disorder.

\section{Acknowledgements}

We are grateful to Dr. S. Baron-Cohen and Prof. Andy Young for reading and commenting on earlier version of this paper.

\section{References}

[1] R.T. Abed, The sexual competition hypothesis for eating disorders, British Journal of Medical Psychology 71 (1998), 525-547.

[2] S. Baron-Cohen, Mindblindness: An essay on autism and theory of mind, MIT Press, Cambridge, 1997.

[3] D. Bickerton, Language and human behaviour, UCL Press, London, 1996.

[4] S. Blackmore, The meme machine, Oxford University Press, Oxford, 1999.

[5] D. Bolton and J. Hill, Mind, meaning and mental disorder: The nature of causal explanations in psychology and psychiatry, Oxford University Press, Oxford, 1996.

[6] J.L. Bradshaw, Human evolution: A neuropsychological perspective, Psychology Press, Hove, 1997.

[7] K. Browne, Divided labours: An evolutionary view of women at work, Weidenfeld \& Nicolson, London, 1998.

[8] D.M. Buss, Evolutionary psychology: The new science of the mind, Allyn \& Bacon, Boston, 1999

[9] M.L. Buttolph, K.E. Peets and A.M. Holland, Obsessivecompulsive disorder symptoms and medication treatment in pregnancy, in: Obsessive-compulsive disorders: Practical management, M.A. Jenike, L. Baer and W.E. Minichiello, eds, Mosby, St. Louis, 1998, pp. 84-96.

[10] R. Carter, Mapping the mind, Weidenfeld \& Nicholson, London, 1998.

[11] N. Chomsky, Rules and representations, Behavioral and Brain Science 3 (1980), 1-61.

[12] H. Cleckley, The mask of sanity, 5th ed, Mosby, St. Louis, 1976.

[13] C.R. Cloninger, Assessment of impulsive-compulsive spectrum of behavior by the seven-factor model of temperament and character, in: Impulsivity and compulsivity, J.M. Oldham, E. Hollander and A.E. Skodol, eds, American Psychiatric Press, Washington, 1996, pp. 59-95.

[14] C.R. Cloninger, D.M. Svarikic and T.R. Przybeck, A psychobiological model of temperament and character, Archives of General Psychiatry 50 (1993), 975-990.

[15] L. Cosmides and J. Tooby, Cognitive adaptations for social exchange, in: The adapted mind: Evolutionary psychology and the generation of culture, J.H. Barkow, L. Cosmides and J. Tooby, eds, Oxford University Press, Oxford, 1992, pp. 163-228. 
[16] M. Daly and M. Wilson, Sex, evolution and behaviour, Wadsworth, Belmont, 1983.

[17] N.H. Dodman, A. Moon-Fenelli, P.A. Mertens, S. Pflueger and D.J. Stein, Veterinery models in OCD, in: ObsessiveCompulsive disorders: diagnosis, etiology, treatment, E. Hollander and D.J. Stein, eds, Marcel Dekker, New York, 1997, pp. 99-143.

[18] S. Dulaney and A.P. Fiske, Cultural rituals and obsessivecompulsive disorder: Is there a common psychological mechanism?, Ethos 22 (1994), 243-283.

[19] A.P. Fiske and N. Haslam, Is obsessive-compulsive disorder a pathology of the human disposition to perform socially meaningful rituals? Evidence of similar content, Journal of Nervous and Mental Disease 185 (1997), 211-222.

[20] J. Fodor, The modularity of mind, MIT Press, Cambridge, 1983.

[21] M.S. Gazzaniga, The mind's past, University of California Press, Berkley, 1998.

[22] P. Gilbert, Defence and safety: Their function in in social behaviour and psychopathology, British Journal of Clinical Psychology 2 (1993), 131-153.

[23] W.D. Hamilton, The genetical evolution of social behaviour I \& II, Journal of Theoretical Biology 7 (1964), 1-52.

[24] R.D. Hare, The Hare psychopathy checklist-revised, MultiHealth Systems, Toronto, 1991.

[25] E. Hollander and L.J. Cohen, Psychobiology and psychopharmacology of compulsive disorders, in: Impulsivity and Compulsivity, J.M. Oldham, E. Hollander and A.E. Skodol, eds, American Psychiatric Press, Washington, 1996, pp. 143-166.

[26] M. Karno and J.M. Golding, Obsessive-compulsive disorder, in: Psychiatric disorders in America: The epidemiologic catchment area study, L.N. Robins and D.A. Regier, eds, Free Press, New York, 1991, pp. 204-219.

[27] H.L. Leonard, E.L. Goldberger, J.L. Rapoport, D.L. Cheslow and S.E. Swedo, Childhood rituals: Normal development or obsessive-compulsive symptoms?, Journal of the American Academy of Child and Adolescent Psychiatry 29 (1990), 1723.

[28] J.F. Lipinski and H.G. Pope, Do 'flashbacks' represent obsessional imagery?, Comprehensive Psychiatry 35 (1994), 245247.

[29] J.J. Lopez-Ibor, Impulse control in obsessive-compulsive disorder: A biopsychopathological approach, Progress in Neuropsychopharmacology and Biological Psychiatry 14 (1990), 709-718.

[30] D.T. Lykken, The antisocial personalities, Lawrence Erlbaum, Hillside, 1995.

[31] I. Marks, Fears, phobias and rituals: Panic, anxiety and their disorders, Oxford University Press, Oxford, 1987.

[32] I.M. Marks and R.M. Nesse, Fear and fitness: An evolutionary analysis of anxiety disorders, Ethology and Sociobiology 15 (1994), 247-261.

[33] M. McGuire and A. Troisi, Darwinian psychiatry, Oxford University Press, Oxford, 1998

[34] S. Mithen, The prehistory of the mind: A search for the origins of art, Religion and science, Thames \& Hudson, London,
1996.

[35] R.M. Nesse and G.C. Williams, Evolution and healing: The new science of Darwinian medicine, Weidenfeld \& Nicholson, London, 1995.

[36] I. Osborn, Tormenting thoughts and secret ritual, Pantheon Books, New York, 1998.

[37] J.V. Penn, J. March and H.L. Leonard, Obsessive-compulsive disorder in children and adolescents, in: Review of Psychiatry, Vol. 16, Section III, L.J. Dickstein, M.B. Riba and J.M. Oldham, eds, American Psychiatric Press, Washington, 1997, pp. 7-28.

[38] S. Pinker, The language instinct, Harper Collins, New York, 1994.

[39] S. Pinker, How the mind works, Allen Lane, Harmondsworth, 1998.

[40] H. Plotkin, Darwin machines and the nature of knowledge, Allen Lane, Harmondsworth, 1994.

[41] H. Plotkin, Evolution in mind: An introduction to evolutionary psychology, Allen Lane, Harmondsworth, 1997.

[42] S. Rachman and P. DeSilva, Abnormal and normal obsessions, Behavioural Research and Therapy 16 (1978), 233248.

[43] S. Rachman and R. Shafran, Cognitive and behavioural features of obsessive-compulsive disorder, in: Obsessivecompulsive disorder: Theory, research and treatment, R.P. Swinson, M.M. Antony, S. Rachman and M.A. Richter, eds, Guilford Press, New York, 1998, pp. 51-78.

[44] J. Rapoport and A. Fiske, The new biology of obsessivecompulsive disorder: Implications for evolutionary psychology, Perspectives in Biology and Medicine 41 (1998), 159179.

[45] J.J. Ratey and C. Johnson, Shadow syndromes, Pantheon Books, New York, 1997

[46] D.R. Rosenberg and M.S. Keshavan, Toward a neurodevelopmental model of obsessive-compulsive disorder, Biological Psychiatry 43 (1998), 623-640.

[47] P.M. Salkovskis, Psychological approaches to the understanding of obsessional problems, in: Obsessional Disorder: Theory, Research and Treatment, R.P. Swinson, M.M. Antony, S. Rachman and M.A. Richter, eds, Guilford Press, New York, 1998, pp. 33-49.

[48] D. Sperber, Explaining culture: A naturalistic approac, Blackwell, Oxford, 1996.

[49] D.J. Stein and J.L. Rapoport, Cross-cultural studies and obsessive-compulsive disorder, CNS Spectrums 1 (1996), 4246.

[50] A. Stevens and J. Price, Evolutionary psychiatry: A new beginning, Routledge, London, 1996.

[51] P.H. Thomsen, From thoughts to obsessions: Obsessivecompulsive disorders in children and adolescents, Jessica Kingsley, London, 1998.

[52] M.M. Weissman, Cross-national epidemiology of obsessivecompulsive disorder, CNS Spectrums 3 (5 suppl) (1998), 6-9.

[53] D.R. Wilson, Evolutionary epidemiology and manic depression, Journal of Medical Psychology 71 (1998), 375-395. 


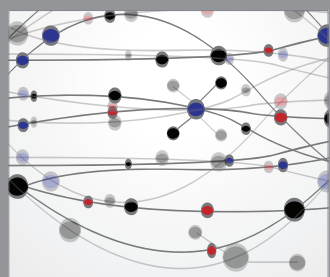

The Scientific World Journal
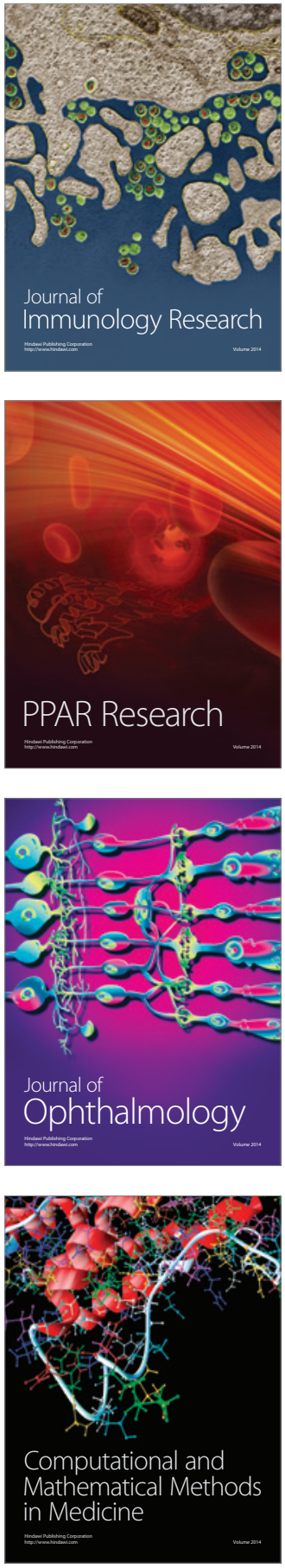

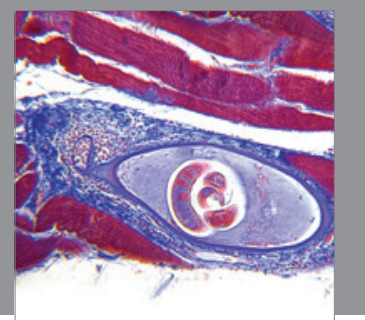

Gastroenterology

Research and Practice


\section{Hindawi}

Submit your manuscripts at

http://www.hindawi.com
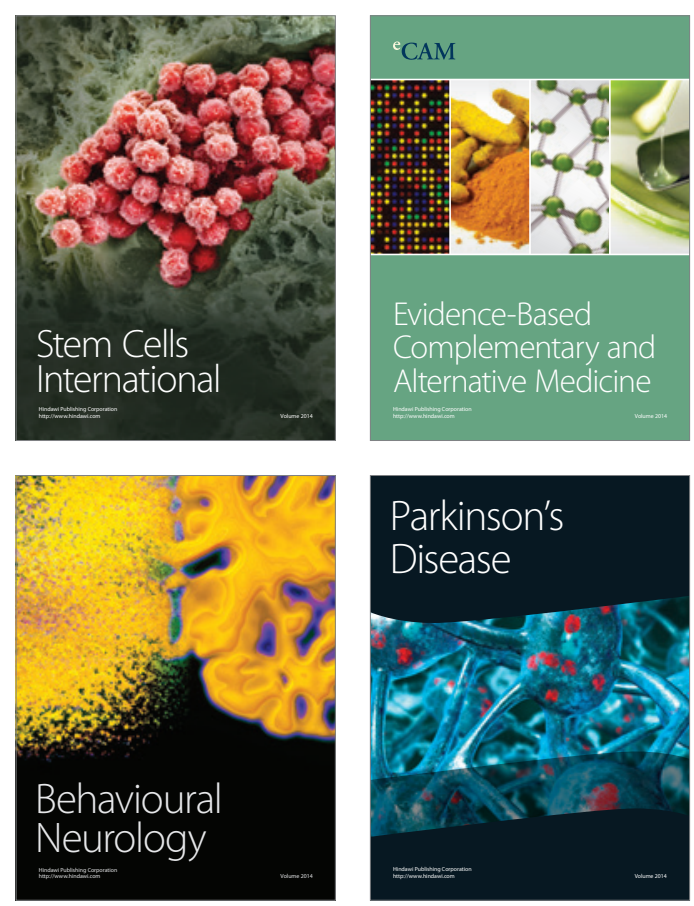

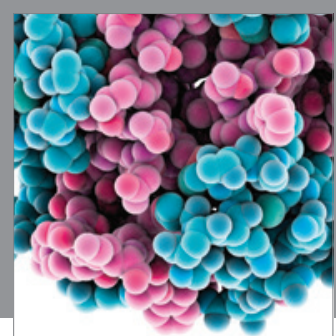

Journal of
Diabetes Research

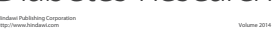



Disease Markers
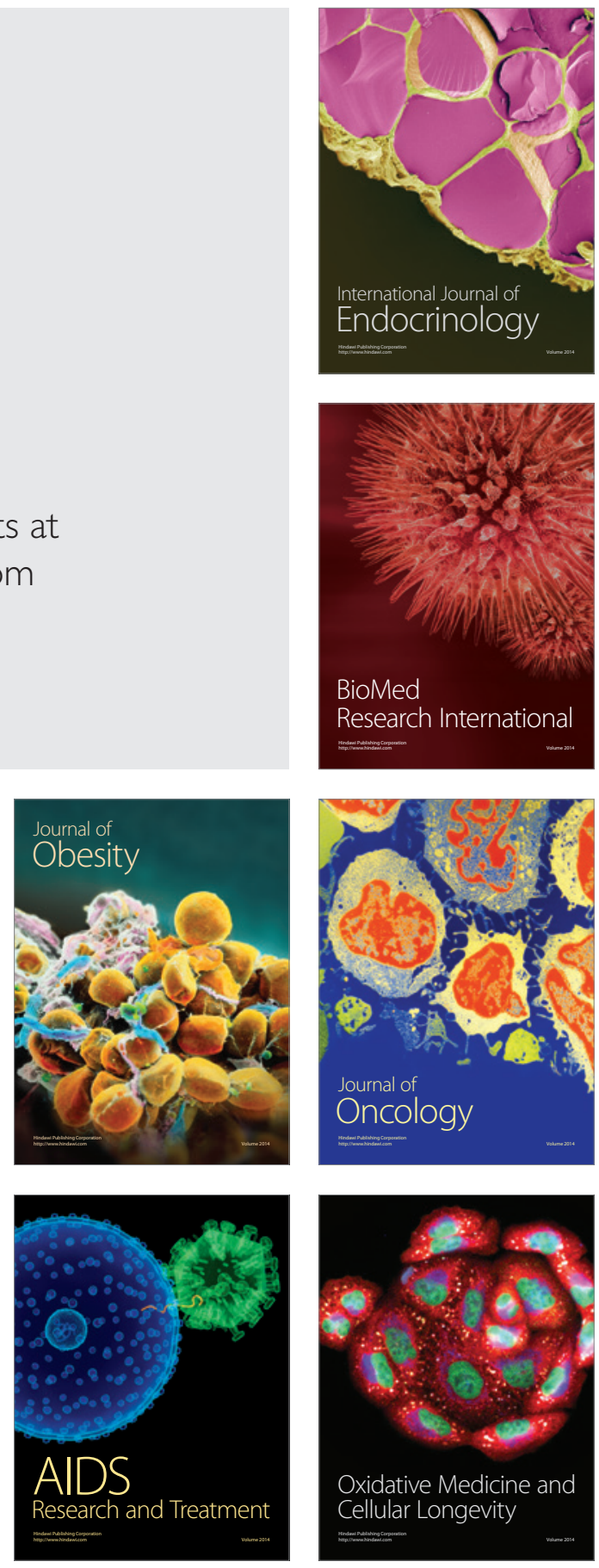